Review

\title{
The Potential Role of the Proteases Cathepsin D and Cathepsin $L$ in the Progression and Metastasis of Epithelial Ovarian Cancer
}

\author{
Md Zahidul Islam Pranjol ${ }^{1, \dagger}$, Nicholas Gutowski ${ }^{1,2}$, Michael Hannemann ${ }^{2}$ \\ and Jacqueline Whatmore $1, \dagger, *$
}

1 Institute of Biomedical and Clinical Science, University of Exeter Medical School, Exeter, Devon EX12LU, UK; E-Mails: Z.Pranjol@exeter.ac.uk (M.Z.I.P.);

N.J.Gutowski@exeter.ac.uk (N.G.)

2 Royal Devon and Exeter NHS Foundation Trust, Exeter, Devon EX2 7JU, UK;

E-Mail: michael.hannemann@nhs.net

$\dagger$ These authors contributed equally to this work.

* Author to whom correspondence should be addressed; E-Mail: J.L.Whatmore@exeter.ac.uk; Tel.: +44-139-226-2944.

Academic Editor: Maria Barbolina

Received: 7 October 2015 / Accepted: 13 November 2015 / Published: 20 November 2015

\begin{abstract}
Epithelial ovarian cancer (EOC) is the leading cause of death from gynecologic malignancies and has a poor prognosis due to relatively unspecific early symptoms, and thus often advanced stage, metastasized cancer at presentation. Metastasis of EOC occurs primarily through the transcoelomic route whereby exfoliated tumor cells disseminate within the abdominal cavity, particularly to the omentum. Primary and metastatic tumor growth requires a pool of proangiogenic factors in the microenvironment which propagate new vasculature in the growing cancer. Recent evidence suggests that proangiogenic factors other than the widely known, potent angiogenic factor vascular endothelial growth factor may mediate growth and metastasis of ovarian cancer. In this review we examine the role of some of these alternative factors, specifically cathepsin D and cathepsin L.
\end{abstract}

Keywords: epithelial ovarian cancer; angiogenesis; metastasis; cathepsin D; cathepsin L 


\section{Introduction}

Epithelial ovarian carcinoma (EOC) is the leading cause of death among gynecological cancers in the western world. Worldwide, approximately 200,000 women are diagnosed with this malignancy, with 125,000 disease related deaths each year [1]. The high mortality of this disease can be explained by late diagnosis at an advanced disease state, with widespread metastasis within the peritoneal cavity [2]. Symptoms are vague at the primary stage, and hence early diagnosis is difficult. Approximately, $70 \%$ of patients are diagnosed with the International Federation of Gynecology and Obstetrics (FIGO) stage III or IV, with a poor five year survival rate. Although the ideal primary cytoreductive surgery and combination chemotherapy with platinum have improved the prognosis of patients with malignant ovarian cancer, the 5-year survival rate remains $\sim 40 \%[3,4]$.

Approximately $90 \%$ of human ovarian cancers are thought to originate from the epithelium [5]. Initially, EOC cells tend to undergo epithelial-to-mesenchymal (EMT) transition which weakens the attachment of epithelial cells to the basement membrane and loosens the intercellular adhesion between neighboring epithelial cells. Loss of expression of E-cadherin correlates with EMT and the acquisition of an invasive phenotype, and cells with low E-cadherin expression have been found in ascites and at metastatic sites [6-8]. As a part of a feedback loop, other cadherins are upregulated in these cells e.g., $\mathrm{N}$-cadherin and P-cadherin [9,10]. These transformed cells are shed as single cells or clusters into ascites or peritoneal fluid and spread through the peritoneum, especially to the omentum [11]. At the omentum, the tumor cells undergo mesenchymal-to-epithelial transition into an epithelial phenotype; a transformation that is vital to allow them to respond to paracrine growth factors and sustain rapid growth [11]. The disseminated cancer cells first interact with the mesothelial layer that covers the omentum. Alpha and beta integrins on both cancer and mesothelial cells have been shown to bind to each other, initiating attachment of cancer cells to the omentum $[12,13]$. This attachment induces upregulation of matrix metalloproteinases (MMPs) 2 and 9 in cancer cells that cleave the extracellular matrix proteins fibronectin, vitronectin and collagens [11]. Early metastasis is well-coordinated via adhesion and proteolysis, which provides a niche for EOCs to establish secondary foci in the omentum by invading the basement membrane.

In order for the secondary tumor to grow and survive, angiogenesis is vital. In the tumor microenvironment the balance between pro- and anti-angiogenic factors favors the proangiogenic process, leading to activation, proliferation and migration of the endothelial cells lining vasculature and excessive genesis of new blood vessels from the existing ones. To date, several proangiogenic factors have been identified to be secreted by EOCs which could potentially drive angiogenesis and metastasis; these include various isoforms of the major pro-angiogenic mediator vascular endothelial growth factor (VEGF) as well as angiopoietin-2, basic fibroblast growth factor (bFGF), heparin-binding EGF-like growth factors (hb-EGF), and cytokines such as interleukins 6 and 8 , and transforming growth factor- $\beta 1$ (TGF $\beta 1)$ [14-17].

Although these factors are now known to be secreted from EOCs, their involvement in inducing metastatic angiogenesis in secondary locations such as the omentum is not fully understood. Indeed, recent evidence suggests that omental metastasis of EOC may primarily occur via non-VEGF dependent pathways [18]. Further to this observation, several other potential proangiogenic factors were identified to be secreted from EOCs. These included cathepsin D (CathD) and cathepsin L (CathL) which were 
shown to induce proangiogenic responses in disease-relevant human omental microvascular endothelial cells [18]. These data suggest that these proteases may play an important role as alternative mediators of metastasis of EOC to the omentum. Since the clinical prevention and treatment of this pathological event is so critical to patient well-being, understanding the function of alternative mechanisms of angiogenesis may be important in targeting future therapies. In this review we aim to summarize the biological and clinical significance of CathD and CathL, initially in physiological cell regulation and then as potential pro-tumorigenic factors regulating progression steps in both primary and metastatic tumors including cell detachment, proliferation and migration, apoptosis and angiogenesis. In particular we will summarize what is known about their role in the etiology of both primary and secondary EOCs.

\section{Cathepsin D}

CathD is a soluble lysosomal aspartic endopeptidase primarily involved in degrading unfolded or non-functional proteins intracellularly. The protein is synthesized in rough endoplasmic reticulum as inactive preprocathepsin $\mathrm{D}(43 \mathrm{kDa})$, which is in turn cleaved and glycosylated to form $52 \mathrm{kDa}$ procathepsin $\mathrm{D}$ ( $\mathrm{pCathD}$ ) containing two N-linked oligosaccharides modified with mannose 6-phosphate (M6P) residues. pCathD is then targeted to intracellular vesicular structures such as lysosomes, endosomes and phagosomes both by M6P receptor (M6PR)-dependent and-independent pathways (reviewed in [19]). The latter pathway of targeting is not fully understood; however the sphingolipid activator precursor protein pro-saponin has been suggested to be involved [20].

Once pCathD enters the late endosome, the low $\mathrm{pH}$ induces its dissociation from M6PR and subsequently the phosphate group is removed. Proteolytic cleavage of propeptide (44aa) on pCathD generates active intermediate enzyme [21]. The propeptide (also known as activation peptide) is essential for the correct folding, activation and delivery of the protein to lysosomes [22,23]. This peptide, which is expressed in, and secreted from, cancer cells, has also been demonstrated to act as a growth factor for tumor cells [24]. The intermediate CathD is further processed by cysteine proteases and autocatalysis to generate mature CathD (48 kDa) containing a heavy chain (34 kDa) and a light chain (14 kDa) [25]. The optimum $\mathrm{pH}$ for CathD activity is 3.5 at which it is highly proteolytically active [26]. However, proteolytic activity has also been reported at neutral $\mathrm{pH}$ in the cytosol of apoptotic cells and in neurofibrillary degeneration $[27,28]$.

\subsection{Physiological Roles of CathD as Both AN Intracellular and Extracellular Protein}

CathD has been shown to play a significant role during fetal development. The lysosomal system matures gradually which correlates with increased CathD levels in all tissues [29]. A reduction of CathD expression or its catalytic activity results in neurodegenerative disorders. CathD knockout mice die shortly after birth and display significant neurodegeneration [29]. Congenital mutations in the CathD gene lead to a reduction in expression and subsequent production of enzymatically inactive protein that results in typical neuronal ceroid lipofuscinoses in dogs and humans [30-35]. Recently, it has been shown that CathD deficiency is associated with Parkinson's disease [36]. Interestingly, increased CathD expression and activity in cardiac cells induces heart failure in postpartum female mice [37]. Higher CathD levels have also been suggested to play an important role in the pathogenesis of autism [38]. 
Several physiological functions of CathD have been suggested based on its proteolytic activity to cleave structural and functional proteins and peptides. These include metabolic degradation of intracellular proteins, activation and degradation of polypeptide hormones and growth factors such as plasminogen, prolactin, endostatin, osteocalcin, thyroglobulin, insulin-like growth factor binding proteins (IGFBP) and secondary lymphoid tissue chemokine (SLC); activation of enzymatic precursors of CathL, CathB and transglutaminase 1; and processing of the enzyme activators and inhibitors prosaposin and cystatin C (reviewed in [19]).

Although CathD is a lysosomal enzyme and its enzyme activity is usually regulated within the acidic compartment of lysosomes, it has been shown to be enzymatically active and biologically relevant extra-lysosomally at cytosolic $\mathrm{pH}$, for instance in the control of apoptosis as discussed later.

Unlike other aspartic endopeptidases, under normal physiological conditions, $\mathrm{pCathD}$ is sequestered to the lysosome and not secreted extracellularly. However, in some conditions, pCathD/CathD escape the normal targeting pathway and are secreted from the cells. Most probably, over-expression of pCathD saturates the limited number of M6PR binding sites available and the protein accumulates in the cytosol, and is subsequently secreted via an as yet unknown mechanism [39]. Indeed pCathD has been found in human, bovine and rat milk and serum and the presence of both pCathD and CathD (34 kDa) was observed in human eccrine sweat and urine [40-43]. CathD in human eccrine sweat was found to be proteolytically active at sweat $\mathrm{pH} 5.5$ [44]. Interestingly, there is increasing evidence that extracellular CathD may act via both proteolytic dependent and independent mechanisms.

\subsection{Expression of CathD in Ovarian Cancer}

In many cancer microenvironments, $\mathrm{pCathD}$ is a major secreted protein. In the last 2 decades, studies have shown increased overexpression and hypersecretion of CathD in numerous cancer types including ovarian cancer, but also in breast cancer, endometrial cancer, lung cancer, malignant glioma, melanoma and prostate cancer (Table 1) [18,45-58]. Early studies investigating ovarian carcinoma suggested that the expression level of CathD was associated with increased cell differentiation and with histological type $[59,60]$. Additionally, numerous immunohistochemistry studies have indicated that enhanced CathD expression is an indicator of malignancy in serous ovarian cancer [61-63], for instance Losch et al. observed that over 70\% of invasive ovarian cancers express CathD [62]. Intriguingly, however, it has also been shown that in ovarian tumors that do express CathD, a high expression level was associated with a favorable survival prognosis [63]. More recently, in an investigation into omental metastasis of ovarian cancer we have observed a significantly higher expression of CathD in the omental lesion of serous ovarian carcinoma compared with omentum from patients with benign ovarian cystadenoma and that high omental mesothelial expression of CathD was associated with poor disease-specific survival (DSS) [64]. This stronger expression of CathD in mesothelial cells was observed close to the metastatic tumor, suggesting a paracrine effect for factors secreted from the tumor cells contributing to the increased CathD expression.

A number of studies have also examined CathD expression in breast cancer. CathD overexpression is correlated with increased risk of clinical metastasis and short survival in breast cancer [45-47] and increased serum pCathD levels were detected in the plasma of patients with metastatic breast 
carcinoma [65]. Additionally, total CathD concentration in breast cancer tissue was much higher than in other tissues including normal mammary cells [66].

Table 1. Involvement of cathepsin D in the stages of tumor progression in different cancer types.

\begin{tabular}{ccccc}
\hline Cancer Type & Metastasis & Invasion & Angiogenesis & References \\
\hline Breast & $\uparrow$ & $\uparrow$ & $\uparrow$ & {$[45-48]$} \\
Ovarian & $\mathrm{ND}$ & $\mathrm{ND}$ & $\uparrow$ & {$[18]$} \\
Prostate & $\uparrow$ & $\uparrow$ & $\downarrow$ & {$[49-51]$} \\
Endometrial & $\mathrm{ND}$ & $\uparrow$ & $\mathrm{ND}$ & {$[58]$} \\
Melanocytic & $\uparrow$ & $\uparrow$ & $\mathrm{ND}$ & {$[53]$} \\
Glioma & $\uparrow$ & $\uparrow$ & $\mathrm{ND}$ & {$[54]$} \\
Lung & $\mathrm{ND}$ & $\uparrow$ & $\mathrm{ND}$ & {$[57]$} \\
\hline
\end{tabular}

$\uparrow$, increase in effects; $\downarrow$, reduction in effects; ND, not determined.

\subsection{Role of CathD in Tumor Progression}

It is now recognized that CathD has a potential role in multiple tumor progression steps, both in its intracellular and extracellular form.

As indicated above, a role for intracellular cytosolic CathD has been identified in apoptosis. Here the lysosomal enzyme is translocated to the cytosol due to lysosomal membrane permeabilization $[28,67,68]$. Subsequently, CathD actively cleaves the BH3-interacting domain (Bid) to form truncated Bid (tBid) which in turn triggers the insertion of Bax into the mitochondrial membrane $[69,70]$, and leads to the release of cytochrome c from mitochondria into the cytosol [71]. Inhibition of enzymatically-active cytosolic CathD, using the inhibitor pepstatin A (pepA), partially delayed apoptosis induced by IFN-gamma or oxidative stress and when pepA was co-microinjected with CathD [68-70]. The role of CathD in inducing apoptosis has also been shown to be associated with caspases; the pan caspase inhibitor Z-VAD-FMK added in combination with pepA, induced a significant reduction in cell death compared to individual inhibitor treatments. This suggested a strong association between caspases and proteolytically active cytosolic CathD [72,73]. Additionally, CathD has been shown to cleave tau protein in vitro at $\mathrm{pH} 7$ [27]. These studies suggest that, intracellularly, CathD is proteolytically active at cytosolic $\mathrm{pH}$. However, this has been contested by other studies indicating that the effect of a mutant CathD, deprived of its catalytic activity, was indistinguishable from that of the normal enzyme [74,75].

Although, a role for intracellular CathD in apoptosis suggests that the protein may be anti-tumorigenic; this is in contrast to the functions observed for extracellular CathD.

For instance CathD is secreted by EOC cancer cell lines (SKOV3 and A2780) [18], is present in the ascites of patients suffering from ovarian cancer (unpublished data), and exogenous CathD induced migration of human omental microvascular endothelial cells; a key step in angiogenesis during omental metastasis [18]. In a separate study pCathD and CathD have been reported to induce proliferation and migration of cancer cells, fibroblasts and endothelial cells in both a proteolytic dependent and independent manner [76] (Figure 1). While secreted pCathD is generally considered to be proteolytically inactive, it has been proposed that the acidic $\mathrm{pH}$ in the tumor microenvironment promotes the conversion of pCathD into mature, biologically active CathD. This was supported by data indicating that $\mathrm{pCathD}$, 
collected from tumor-conditioned media, became auto-activated if the $\mathrm{pH}$ was lowered and was subsequently able to degrade ECM proteins and release growth factors such as bFGF $[48,77,78]$, steps important for cancer cells to invade surrounding tissue [79].

There is evidence that CathD may induce mitogenic responses via both proteolytic-dependent and-independent mechanisms. Both the wild type and mutant form of CathD were shown to induce fibroblast proliferation via a mechanism whereby they acted as a protein ligand [80]. In the latter study, the authors demonstrated an interaction between M6PR and pCathD. Co-incubation with excess M6P partially prevented fibroblast proliferation. Although an unknown receptor molecule has been suggested to be involved, the identity of this potential receptor has not yet been resolved.

CathD actions on tumor growth were further reported in studies showing that 3Y1-Ad12 rat tumor cells transfected with human CathD cDNA grew more rapidly at low or high cell densities in vitro and presented an increased experimental metastatic potential in vivo [81-83]. Additionally, both wild-type and mutated (Asn 231, proteolytically inactive) CathD stimulated proliferation of 3Y1-Ad12 cells embedded in Matrigel or collagen 1 matrices, colony formation in soft agar and tumor growth in athymic nude mice $[84,85]$. Again, an unknown receptor, other than M6PR, was suggested to be involved in CathD mediated cell growth as no inhibition of cell outgrowth was observed when excess M6P was added, suggesting that M6P did not compete with CathD interacting with M6PR. In the same study the propeptide (27-44aa) of pCathD was found not to be mitogenic, contradicting studies which found otherwise [24,66,86-89].

The role of CathD has also been extensively studied in human primary breast cancer. Upregulation of CathD expression was observed in estrogen receptor (ER) positive breast cancer cell lines treated with estrogen [90]. In vitro experiments with the MCF7 cell line supported these data and revealed that $\mathrm{pCathD/CathD} \mathrm{were} \mathrm{overexpressed} \mathrm{and} \mathrm{hyper-secreted} \mathrm{from} \mathrm{these} \mathrm{cells} \mathrm{into} \mathrm{the} \mathrm{media.} \mathrm{Further} \mathrm{studies}$ have reported that as a mitogen $\mathrm{pCathD}$ acts as a protein ligand rather than enzymatically and that purified pCathD from MCF-7 breast cancer cells stimulated MCF-7 cell growth on plastic via an autocrine mechanism [91]. Intriguingly, CathD has also been shown to selectively degrade macrophage inflammatory protein (MIP)-1 $\alpha$ (CCL3), MIP-1 $\beta$ (CCL4), and SLC (CCL21) that, in turn, may affect the generation of the anti-tumoral immune response, the migration of human breast cancer cells, or both processes [92].

In recent years studies have emerged that suggest that CathD can induce angiogenesis in vivo and in vitro. In vivo, overexpression of CathD in xenografts in an athymic mice model correlated with increased vascular density. The number of microvessels was significantly increased by 1.5 -fold and 1.9-fold in the CathD and CathD-Asn 231(proteolytically inactive) groups respectively, suggesting that CathD induces angiogenic effects via an unknown mechanism other than its proteolytic activity [85].

CathD has also been shown to induce blood vessel formation in the chick chorioallantoic membrane (CAM) model [93] and a role for CathD in angiogenesis was further illustrated by the observation that migration of human umbilical vein endothelial cells (HUVECs) and in vitro angiogenic tube formation were increased when cells were treated with active pure CathD. CathD was proteolytically active in these experiments as complete inhibition of angiogenesis, tube formation and migration was achieved by addition of pepA [93]. Proteolytically active CathD has also been suggested to induce angiogenesis in breast cancer by cleaving and releasing ECM-bound pro-angiogenic bFGF [48]. 


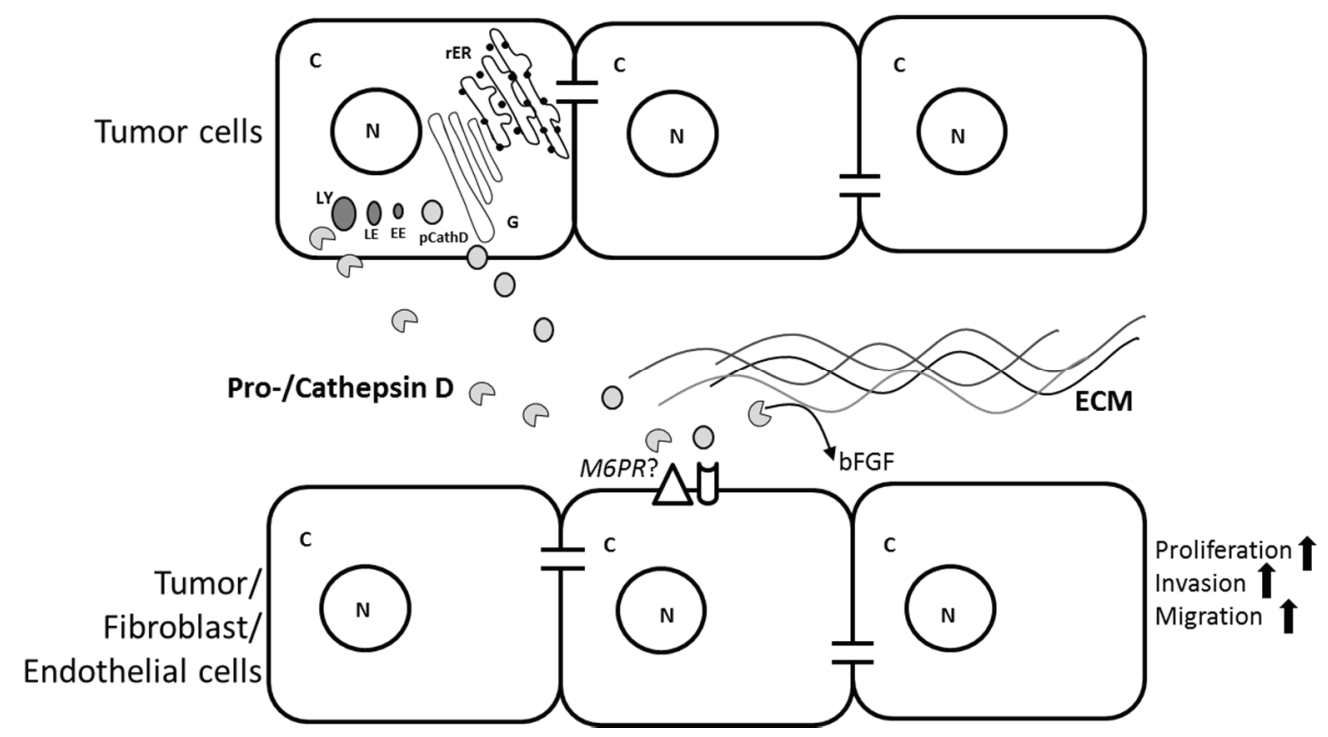

Figure 1. Potential roles of tumor cell-secreted procathepsin/cathepsin D (pCathD/CathD) on extracellular matrix (ECM), tumor, fibroblast and endothelial cells in the tumor microenvironment. pCathD is synthesized and processed in the rough endoplasmic reticulum (rER) and Golgi bodies (G), and subsequently transported to early endosome (EE), late endosome (LE) and finally lysosome (LY). Overexpressed pCathD/CathD is secreted into the extracellular space by tumor cells. Mature CathD cleaves ECM and releases basic fibroblast growth factor (bFGF) that may induce angiogenesis. Both pCathD and CathD induce tumor cell proliferation, and hence invasion via an autocrine mechanism. CathD induces proliferation of fibroblasts and migration of endothelial cells. Mannose-6-phosphate receptor (M6PR) may be involved in inducing the proliferative effects. $\mathrm{C}$ and $\mathrm{N}$ denote cytoplasm and nucleus, respectively.

In contrast it has also been suggested that CathD activity may be anti-angiogenic For instance, $\mathrm{pCathD}$ secreted by prostate cancer cells was shown to have a possible role in generating angiostatin via proteolysis - a specific inhibitor of angiogenesis in vitro as well as in vivo [51].

\section{Cathepsin L}

CathL is a lysosomal ubiquitous cysteine proteinase that plays an important role in degrading endocytosed proteins as well as intracellular proteins $[94,95]$. CathL is translated as preprocathepsin $\mathrm{L}$ (ppCathL), processed into procathepsin $\mathrm{L}$ (pCathL) in the rough endoplasmic reticulum with a molecular mass of $30 \mathrm{kDa}$ and a two-chain form with molecular masses $25 \mathrm{kDa}$ and $5 \mathrm{kDa}$ [96-99], and then transported to endosome/lysosomes in M6PR pathway [100]. It has been reported that 1, 10-phenanthrolin and pepstatin partially inhibited the processing of the proenzyme form of CathL to the mature enzyme and it has been speculated that metallo-proteinases or an aspartic protease such as CathD are involved in the proteolysis-mediated activation of CathL in the lysosome [101,102]. CathL can also be produced by autocatalysis of $\mathrm{pCathL}$ at $\mathrm{pH} 3$ as demonstrated in an in vitro study of pCathL collected into conditioned medium of cultured murine fibroblasts [103]. 


\subsection{Physiological Roles of CathL}

The key functional role of CathL is to degrade proteins in lysosomes and it has been shown to be highly active at physiological pH 5.5-6 in lysosomes in several studies [103,104]. However, it is also known that CathL is secreted in different forms into the extracellular space in both physiological and pathological conditions, and retains its function as a protease. In physiology, CathL has been shown to degrade the Ii peptide of major histocompatibility complex II (MHC II) that in turn allows peptides derived from the proteolytic degradation of foreign or self-proteins to then bind to class II molecules and appear on the cell surface [105]. Interestingly, it has been shown that CathL is essential for MHC II mediated antigen presentation in cortical thymic epithelial cells but not in bone marrow-derived antigen-presenting cells in vivo. This was reflected in CathL deficient mice with a reduction in CD4+ T cells [105]. CathL has also been shown to degrade and process MHC II molecule-mediated antigen presentation [106]. CathL is essential for epidermal homeostasis and regular hair follicle morphogenesis and cycling; indeed, CathL-deficient mice develop periodic hair loss and epidermal hyperplasia, acanthosis, and hyperkeratosis [107].

It has also been reported that CathL null mice showed reduced bone mass compared to wild type mice suggesting a role for CathL in bone remodeling. CathL null mice showed significant reduction in bone volume in travecular, but not cortical, bone compared to wild type. Bone loss was exacerbated in null mice (compared to wild type) following ovariectomy suggesting that CathL is stimulated by external stimuli (e.g., estrogen) and is likely to play a role in controlling bone turnover during normal development and in pathological states [108].

\subsection{Cathepsin L Secretion}

A secreted form of CathL was first identified as a major secreted protein from a transformed mouse fibroblast cell line [103]. However, the mechanism of CathL secretion is still a mystery. pCathL has only one single chain carbohydrate, and hence it has low affinity for M6PR [109-111]. This observation suggests that not all CathL binds to M6PR and therefore is secreted from the cell by default protein trafficking [100]. M6PR saturation, downregulation or redistribution to the plasma membrane has also been suggested [112,113]. Interestingly, hypoxia was shown to induce secretion of CathL from the murine fibrosarcoma cell line KHT-LP1 which may accelerate the metastatic process in these cells [114]. CathL has also been shown to be protective against bacterial infection in airways of mice [115].

\subsection{Expression of CathL in Ovarian Cancer}

CathL has been linked to tumor invasion and metastasis, particularly by degrading ECM components such as proteoglycans, aggrecan, elastin, laminin, fibronectin and collagens I, II, IX, XI [116-121]. In ovarian cancer an increased level of secreted CathL was observed in the sera of epithelial malignant EOC patients compared to those with benign ovarian tumors and normal ovarian tissue [122,123]. These studies also showed that there was a significant increase in the tumor expression of CathL mRNA levels which correlated with its protein levels in serum. CathL has been suggested to be involved in the invasion and metastasis of EOC, and hence maybe a marker of advanced stage ovarian cancer [123]. This is supported by our own data demonstrating that the endothelium of vessels within omentum hosting 
metastatic ovarian high-grade serous carcinoma expressed significantly increased CathL in vivo compared with omentum from control patients with benign ovarian cystadenoma [64].

\subsection{Role of CathL in Tumor Progression}

CathL may play a role in the proliferation of ovarian cancer cells, although the data are contradictory. CathL had little effect on cell growth and proliferation of the A2780 ovarian cancer cell line [123], whereas downregulation of CathL significantly inhibited the proliferative and invasive capability of SKOV3 ovarian cancer cells [124].

Over-expression of CathL has been linked to metastasis following ras transformation of NIH/3T3 cells [125] and non-metastatic melanoma cells were converted to a metastatic state by over-expression of CathL [126]. An extra-lysosomal role for CathL has been suggested in human and murine melanoma cells in the context of metastasis [127]. Recently, it was shown that CathL is involved in B16F10 melanoma cell invasion, particularly through cell migratory influences. There was approximately a $70 \%$ reduction in CathL anti-sense clone invasion and migration compared to control after $24 \mathrm{~h}$. However, when CathL-induced proliferation was tested in these cells, no difference was found between the rate of proliferation of antisense cell and control cell colonies. Overall, the results suggested that secreted CathL has direct effects on cell motility and contributes, via its proteolytic action, to the active invasion of melanoma cells [128]. CathL-induced pancreatic cancer cell invasion was also observed in RT2 mice. CathL null mice had a significant reduction in tumor volume and invasion, suggesting extracellular proteolytic activity. In contrast to the melanoma cell study discussed above, the latter work demonstrated significant proliferative effects of CathL on pancreatic cancer cells, with a 58\% decrease in proliferation in CathL knockout cells [129].

Recently, CathL derived from skeletal muscle cells transfected with bFGF has been shown to promote migration of HUVECs [130]. Cell migration was examined in the presence of the cell impermeable CathL-proteolytic activity inhibitor Z-Phe-Tyr-Cho and CathL for 12 hours. The data revealed a significant reduction in HUVEC migration, suggesting that CathL influences cell migration in a manner dependent on its proteolytic activity. Subsequently, CathL was found to activate c-Jun N-terminal kinase (JNK) in migratory HUVECs [130]. However, the exact role of CathL in activating the JNK pathway has not been elucidated.

Evidence for a role for CathL in angiogenesis is also contradictory. Recently SKOV3 and A2780 EOC cells were shown to secrete CathL. Exogenous addition of CathL to human omental microvascular endothelial cells used as an in vitro model of omental angiogenesis, induced migration and in vitro tube structure formation [18] (Figure 2). Together, these data suggest that CathL may trigger a proangiogenic phenotype in these endothelial cells.

In contrast to the pro-angiogenic role of CathL discussed above, both secreted and intracellular CathL has been shown to release endostatin, a potent inhibitor of angiogenesis, by cleaving ECM collagen [131]. Since the tumor microenvironment provides an acidic milieu, CathL can efficiently cleave collagen even outside the cells. However, in other studies, CathL had no effect on angiogenesis. For instance, Gocheva et al. (2006) demonstrated that CathL had no significant effects on microvascular density in pancreatic cancer in mice [129]. When evaluating the role of CathL in angiogenic switching in homozygous cathepsin knockout RT2 mice compared to control mice, the authors found that there was 
no significant effect on the development of these precursor lesions, suggesting that CathL did not contribute to angiogenic switching. However, other cathepsins, i.e. cathepsins B and S, were found to be very important in inducing angiogenesis in the same study [129].

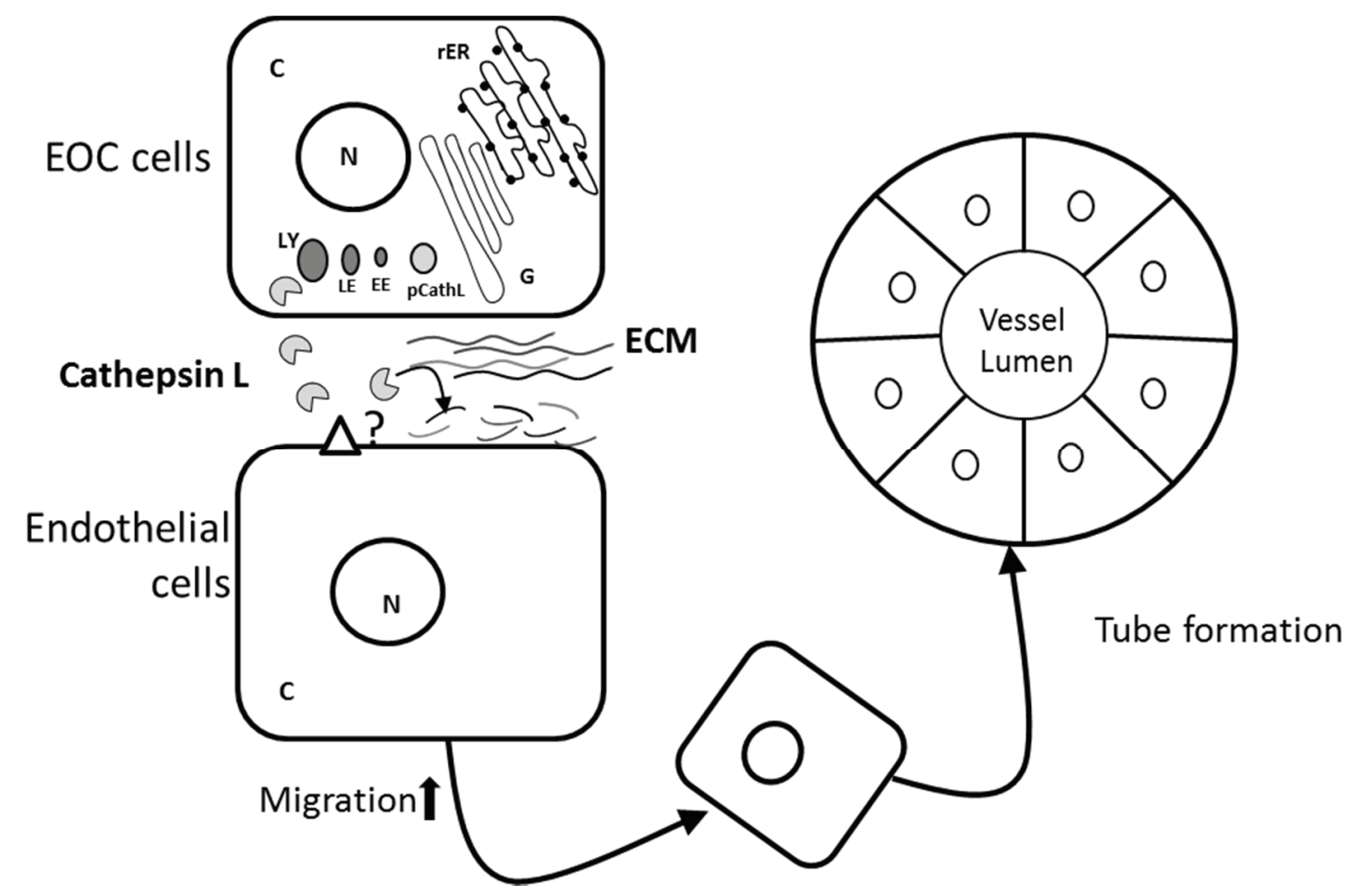

Figure 2. Potential proangiogenic role of cathepsin L in EOC. Procathepsin L (pCathL) is synthesised and processed in the rough endoplasmic reticulum (rER) and Golgi bodies $(\mathrm{G})$, and subsequently translocated to early endosome (EE), late endosome (LE) and finally lysosome (LE). Mature CathL is secreted by epithelial ovarian cancer (EOC) cells and induces migration in endothelial cells via an unknown receptor. Tumor-secreted CathL may also degrade extra cellular matrix (ECM) components, facilitating new vessel formation.

Intriguingly, endothelial progenitor cells (EPCs) have been reported to produce CathL which in turn induces angiogenesis. Urbich et al. showed that EPCs were able to stimulate neovascularization and blood flow in the ischemic murine hind leg after injection into the affected leg [132]. These EPCs displayed significantly enhanced expression of CathL compared to mature endothelial cells as revealed by mRNA array analysis. It has been suggested that in the neovascularization process CathL activity may be extra- or pericellular. Indeed, mature CathL has been shown to maintain its proteolytic activity in the extracellular environment at neutral $\mathrm{pH}$ by the chaperone action of a $\mathrm{p} 41$ splice variant of the MHC class II-associated invariant chain [133], which also is strongly expressed in EPCs [132]. Such activity may facilitate EPC invasion and neovascularization and, interestingly, CathL deficient mice suffered from impaired neovascularization. Furthermore, mice treated with CathL-deficient bone marrow cells demonstrated a significant reduction in angiogenesis [132]. These data are supported by the observation that CathL expressed in EPCs cells plays a critical role in intraocular angiogenesis [134]. However, although CathL has been suggested to induce angiogenesis in these studies, its mechanism of action has not been elucidated. 


\section{Conclusions}

Proteases such as CathD and CathL had long been known for their intracellular protein-degrading activities. However, a key role in cancer biology is now recognized, particularly their proteolytic function in ECM breakdown and thus facilitation of invasion. Interestingly, it is now becoming accepted that these proteases may also promote tumorigenesis and metastasis via non-proteolytic actions, although there is still a relative lack of understanding regarding their receptors and downstream intracellular effectors and signaling pathways. In EOC the urgent need to develop effective therapeutic approaches to improve patient outcomes highlights the importance of better understanding the role of key factors, such as the cathepsins, in driving ovarian tumor progression and metastasis in order to identify potential molecular therapeutic targets.

\section{Acknowledgments}

Our previous (cited studies) and work are supported by FORCE Cancer Charity UK.

\section{Author Contributions}

Md Zahidul Islam Pranjol and Jacqueline Whatmore wrote and reviewed the paper. Nicholas Gutowski and Michael Hannemann reviewed the paper.

\section{Conflicts of Interest}

The authors declare no conflict of interest.

\section{References}

1. Parkin, D.M.; Bray, F.; Ferlay, J.; Pisani, P. Global cancer statistics, 2002. CA Cancer J. Clin. 2005, 55, 74-108.

2. Buy, J.N.; Moss, A.A.; Ghossain, M.A.; Sciot, C.; Malbec, L.; Vadrot, D.; Paniel, B.J.; Decroix, Y. Peritoneal implants from ovarian tumors: Ct findings. Radiology 1988, 169, 691-694.

3. Johnatty, S.E.; Beesley, J.; Paul, J.; Fereday, S.; Spurdle, A.B.; Webb, P.M.; Byth, K.; Marsh, S.; McLeod, H.; Group, A.S.; et al. Abcb1 (Mdr 1) polymorphisms and progression-free survival among women with ovarian cancer following paclitaxel/carboplatin chemotherapy. Clin. Cancer Res. 2008, 14, 5594-5601.

4. Deraco, M.; Baratti, D.; Laterza, B.; Balestra, M.R.; Mingrone, E.; Macri, A.; Virzi, S.; Puccio, F.; Ravenda, P.S.; Kusamura, S. Advanced cytoreduction as surgical standard of care and hyperthermic intraperitoneal chemotherapy as promising treatment in epithelial ovarian cancer. Eur. J. Surg. Oncol. 2011, 37, 4-9.

5. Ahmed, N.; Thompson, E.W.; Quinn, M.A. Epithelial-mesenchymal interconversions in normal ovarian surface epithelium and ovarian carcinomas: An exception to the norm. J. Cell. Physiol. 2007, 213, 581-588.

6. Huber, M.A.; Kraut, N.; Beug, H. Molecular requirements for epithelial-mesenchymal transition during tumor progression. Curr. Opin. Cell Biol. 2005, 17, 548-558. 
7. Cavallaro, U.; Christofori, G. Cell adhesion and signalling by cadherins and ig-cams in cancer. Nat. Rev. Cancer 2004, 4, 118-132.

8. Kalluri, R.; Weinberg, R.A. The basics of epithelial-mesenchymal transition. J. Clin. Investig. 2009, 119, 1420-1428.

9. Patel, I.S.; Madan, P.; Getsios, S.; Bertrand, M.A.; MacCalman, C.D. Cadherin switching in ovarian cancer progression. Int. J. Cancer 2003, 106, 172-177.

10. Hudson, L.G.; Zeineldin, R.; Stack, M.S. Phenotypic plasticity of neoplastic ovarian epithelium: Unique cadherin profiles in tumor progression. Clin. Exp. Metastasis 2008, 25, 643-655.

11. Lengyel, E. Ovarian cancer development and metastasis. Am. J. Pathol. 2010, 177, 1053-1064.

12. Strobel, T.; Cannistra, S.A. Betal-integrins partly mediate binding of ovarian cancer cells to peritoneal mesothelium in vitro. Gynecol. Oncol. 1999, 73, 362-367.

13. Slack-Davis, J.K.; Atkins, K.A.; Harrer, C.; Hershey, E.D.; Conaway, M. Vascular cell adhesion molecule-1 is a regulator of ovarian cancer peritoneal metastasis. Cancer Res. 2009, 69, 1469-1476.

14. Van der Bilt, A.R.; van der Zee, A.G.; de Vries, E.G.; de Jong, S.; Timmer-Bosscha, H.; ten Hoor, K.A.; den Dunnen, W.F.; Hollema, H.; Reyners, A.K. Multiple vegf family members are simultaneously expressed in ovarian cancer: A proposed model for bevacizumab resistance. Curr. Pharm. Des. 2012, 18, 3784-3792.

15. Lin, Z.; Liu, Y.; Sun, Y.; He, X. Expression of Ets-1, Ang-2 and maspin in ovarian cancer and their role in tumor angiogenesis. J. Exp. Clin. Cancer Res. 2011, 30, 31-37.

16. Tanaka, Y.; Miyamoto, S.; Suzuki, S.O.; Oki, E.; Yagi, H.; Sonoda, K.; Yamazaki, A.; Mizushima, H.; Maehara, Y.; Mekada, E.; et al. Clinical significance of heparin-binding epidermal growth factor-like growth factor and a disintegrin and metalloprotease 17 expression in human ovarian cancer. Clin. Cancer Res. Off. J. Am. Assoc. Cancer Res. 2005, 11, 4783-4792.

17. Toutirais, O.; Chartier, P.; Dubois, D.; Bouet, F.; Leveque, J.; Catros-Quemener, V.; Genetet, N. Constitutive expression of Tgf-beta1, interleukin- 6 and interleukin- 8 by tumor cells as a major component of immune escape in human ovarian carcinoma. Eur. Cytokine Netw. 2003, 14, $246-255$.

18. Winiarski, B.K.; Wolanska, K.I.; Rai, S.; Ahmed, T.; Acheson, N.; Gutowski, N.J.; Whatmore, J.L. Epithelial ovarian cancer-induced angiogenic phenotype of human omental microvascular endothelial cells may occur independently of vegf signaling. Transl. Oncol. 2013, 6, 703-714.

19. Benes, P.; Vetvicka, V.; Fusek, M. Cathepsin D-Many functions of one aspartic protease. Crit. Rev. Oncol. Hematol. 2008, 68, 12-28.

20. Gopalakrishnan, M.M.; Grosch, H.W.; Locatelli-Hoops, S.; Werth, N.; Smolenova, E.; Nettersheim, M.; Sandhoff, K.; Hasilik, A. Purified recombinant human prosaposin forms oligomers that bind procathepsin D and affect its autoactivation. Biochem. J. 2004, 383, 507-515.

21. Laurent-Matha, V.; Derocq, D.; Prebois, C.; Katunuma, N.; Liaudet-Coopman, E. Processing of human cathepsin $\mathrm{D}$ is independent of its catalytic function and auto-activation: Involvement of cathepsins L and B. J. Biochem. 2006, 139, 363-371.

22. Takeshima, H.; Sakaguchi, M.; Mihara, K.; Murakami, K.; Omura, T. Intracellular targeting of lysosomal cathepsin D in cos cells. J. Biochem. 1995, 118, 981-988.

23. Yasuda, Y.; Tsukuba, T.; Okamoto, K.; Kadowaki, T.; Yamamoto, K. The role of the cathepsin E propeptide in correct folding, maturation and sorting to the endosome. J. Biochem. 2005, 138, $621-630$. 
24. Vetvicka, V.; Vetvickova, J.; Fusek, M. Role of procathepsin D activation peptide in prostate cancer growth. Prostate 2000, 44, 1-7.

25. Gieselmann, V.; Hasilik, A.; von Figura, K. Processing of human cathepsin D in lysosomes in vitro. J. Biol. Chem. 1985, 260, 3215-3220.

26. Yoshinari, M.; Taurog, A. Lysosomal digestion of thyroglobulin: Role of cathepsin D and thiol proteases. Endocrinology 1985, 117, 1621-1631.

27. Kenessey, A.; Nacharaju, P.; Ko, L.W.; Yen, S.H. Degradation of tau by lysosomal enzyme cathepsin D: Implication for alzheimer neurofibrillary degeneration. J. Neurochem. 1997, 69, 2026-2038.

28. Roberg, K.; Johansson, U.; Ollinger, K. Lysosomal release of cathepsin D precedes relocation of cytochrome $\mathrm{C}$ and loss of mitochondrial transmembrane potential during apoptosis induced by oxidative stress. Free Radic. Biol. Med. 1999, 27, 1228-1237.

29. Kageyama, T.; Tatematsu, M.; Ichinose, M.; Yahagi, N.; Miki, K.; Moriyama, A.; Yonezawa, S. Development-dependent expression of cathepsins D and e in various rat tissues, with special reference to the high expression of cathepsin E in fetal liver. Zool. Sci. 1998, 15, 517-523.

30. Tyynela, J.; Sohar, I.; Sleat, D.E.; Gin, R.M.; Donnelly, R.J.; Baumann, M.; Haltia, M.; Lobel, P. A mutation in the ovine cathepsin $\mathrm{D}$ gene causes a congenital lysosomal storage disease with profound neurodegeneration. EMBO J. 2000, 19, 2786-2792.

31. Tyynela, J.; Sohar, I.; Sleat, D.E.; Gin, R.M.; Donnelly, R.J.; Baumann, M.; Haltia, M.; Lobel, P. Congenital ovine neuronal ceroid lipofuscinosis - A cathepsin D deficiency with increased levels of the inactive enzyme. Eur. J. Paediatr. Neurol. 2001, 5, 43-45.

32. Steinfeld, R.; Reinhardt, K.; Schreiber, K.; Hillebrand, M.; Kraetzner, R.; Bruck, W.; Saftig, P.; Gartner, J. Cathepsin d deficiency is associated with a human neurodegenerative disorder. Am. J. Hum. Genet. 2006, 78, 988-998.

33. Fritchie, K.; Siintola, E.; Armao, D.; Lehesjoki, A.E.; Marino, T.; Powell, C.; Tennison, M.; Booker, J.M.; Koch, S.; Partanen, S.; et al. Novel mutation and the first prenatal screening of cathepsin D deficiency (Cln10). Acta Neuropathol. 2009, 117, 201-208.

34. Siintola, E.; Partanen, S.; Stromme, P.; Haapanen, A.; Haltia, M.; Maehlen, J.; Lehesjoki, A.E.; Tyynela, J. Cathepsin d deficiency underlies congenital human neuronal ceroid-lipofuscinosis. Brain 2006, 129, 1438-1445.

35. Awano, T.; Katz, M.L.; O’Brien, D.P.; Taylor, J.F.; Evans, J.; Khan, S.; Sohar, I.; Lobel, P.; Johnson, G.S. A mutation in the cathepsin D gene (CTSD) in american bulldogs with neuronal ceroid lipofuscinosis. Mol. Genet. Metab. 2006, 87, 341-348.

36. Cullen, V.; Lindfors, M.; Ng, J.; Paetau, A.; Swinton, E.; Kolodziej, P.; Boston, H.; Saftig, P.; Woulfe, J.; Feany, M.B.; et al. Cathepsin D expression level affects alpha-synuclein processing, aggregation, and toxicity in vivo. Mol. Brain 2009, doi:10.1186/1756-6606-2-5.

37. Hilfiker-Kleiner, D.; Kaminski, K.; Podewski, E.; Bonda, T.; Schaefer, A.; Sliwa, K.; Forster, O.; Quint, A.; Landmesser, U.; Doerries, C.; et al. A cathepsin D-cleaved $16 \mathrm{kda}$ form of prolactin mediates postpartum cardiomyopathy. Cell 2007, 128, 589-600.

38. Sheikh, A.M.; Li, X.; Wen, G.; Tauqeer, Z.; Brown, W.T.; Malik, M. Cathepsin D and apoptosis related proteins are elevated in the brain of autistic subjects. Neuroscience 2010, 165, 363-370. 
39. Mathieu, M.; Vignon, F.; Capony, F.; Rochefort, H. Estradiol down-regulates the mannose-6phosphate/insulin-like growth factor-ii receptor gene and induces cathepsin-D in breast cancer cells: A receptor saturation mechanism to increase the secretion of lysosomal proenzymes. Mol. Endocrinol. 1991, 5, 815-822.

40. Vetvicka, V.; Vagner, J.; Baudys, M.; Tang, J.; Foundling, S.I.; Fusek, M. Human breast milk contains procathepsin D-Detection by specific antibodies. Biochem. Mol. Biol. Int. 1993, 30, 921-928.

41. Larsen, L.B.; Petersen, T.E. Identification of five molecular forms of cathepsin D in bovine milk. Adv. Exp. Med. Biol. 1995, 362, 279-283.

42. Benes, P.; Koelsch, G.; Dvorak, B.; Fusek, M.; Vetvicka, V. Detection of procathepsin D in rat milk. Comp. Biochem. Physiol. B Biochem. Mol. Biol. 2002, 133, 113-118.

43. Zuhlsdorf, M.; Imort, M.; Hasilik, A.; von Figura, K. Molecular forms of beta-hexosaminidase and cathepsin D in serum and urine of healthy subjects and patients with elevated activity of lysosomal enzymes. Biochem. J. 1983, 213, 733-740.

44. Baechle, D.; Flad, T.; Cansier, A.; Steffen, H.; Schittek, B.; Tolson, J.; Herrmann, T.; Dihazi, H.; Beck, A.; Mueller, G.A.; et al. Cathepsin d is present in human eccrine sweat and involved in the postsecretory processing of the antimicrobial peptide dcd-11. J. Biol. Chem. 2006, 281, 5406-5415.

45. Rochefort, H. Cathepsin d in breast cancer: A tissue marker associated with metastasis. Eur. J. Cancer 1992, 28, 1780-1783.

46. Ferrandina, G.; Scambia, G.; Bardelli, F.; Benedetti Panici, P.; Mancuso, S.; Messori, A. Relationship between cathepsin-D content and disease-free survival in node-negative breast cancer patients: A meta-analysis. Br. J. Cancer 1997, 76, 661-666.

47. Foekens, J.A.; Look, M.P.; Bolt-de Vries, J.; Meijer-van Gelder, M.E.; van Putten, W.L.; Klijn, J.G. Cathepsin-D in primary breast cancer: Prognostic evaluation involving 2810 patients. Br. J. Cancer 1999, 79, 300-307.

48. Briozzo, P.; Badet, J.; Capony, F.; Pieri, I.; Montcourrier, P.; Barritault, D.; Rochefort, H. Mcf7 mammary cancer cells respond to bfgf and internalize it following its release from extracellular matrix: A permissive role of cathepsin D. Exp. Cell Res. 1991, 194, 252-259.

49. Chen, L.; Li, H.; Liu, W.; Zhu, J.; Zhao, X.; Wright, E.; Cao, L.; Ding, I.; Rodgers, G.P. Olfactomedin 4 suppresses prostate cancer cell growth and metastasis via negative interaction with cathepsin D and SDF-1. Carcinogenesis 2011, 32, 986-994.

50. Konno, S.; Cherry, J.P.; Mordente, J.A.; Chapman, J.R.; Choudhury, M.S.; Mallouh, C.; Tazaki, H. Role of cathepsin D in prostatic cancer cell growth and its regulation by brefeldin A. World J. Urol. 2001, 19, 234-239.

51. Morikawa, W.; Yamamoto, K.; Ishikawa, S.; Takemoto, S.; Ono, M.; Fukushi, J.; Naito, S.; Nozaki, C.; Iwanaga, S.; Kuwano, M. Angiostatin generation by cathepsin D secreted by human prostate carcinoma cells. J. Biol. Chem. 2000, 275, 38912-38920.

52. Losch, A.; Kohlberger, P.; Gitsch, G.; Kaider, A.; Breitenecker, G.; Kainz, C. Lysosomal protease cathepsin D is a prognostic marker in endometrial cancer. Br. J. Cancer 1996, 73, 1525-1528.

53. Zhu, L.; Wada, M.; Usagawa, Y.; Yasukochi, Y.; Yokoyama, A.; Wada, N.; Sakamoto, M.; Maekawa, T.; Miyazaki, R.; Yonenaga, E.; et al. Overexpression of cathepsin D in malignant melanoma. Fukuoka Igaku Zasshi 2013, 104, 370-375. 
54. Fukuda, M.E.; Iwadate, Y.; Machida, T.; Hiwasa, T.; Nimura, Y.; Nagai, Y.; Takiguchi, M.; Tanzawa, H.; Yamaura, A.; Seki, N. Cathepsin d is a potential serum marker for poor prognosis in glioma patients. Cancer Res. 2005, 65, 5190-5194.

55. Rochefort, H.; Garcia, M.; Glondu, M.; Laurent, V.; Liaudet, E.; Rey, J.M.; Roger, P. Cathepsin D in breast cancer: Mechanisms and clinical applications, a 1999 overview. Clin. Chim. Acta 2000, 291, 157-170.

56. Pruitt, F.L.; He, Y.; Franco, O.E.; Jiang, M.; Cates, J.M.; Hayward, S.W. Cathepsin d acts as an essential mediator to promote malignancy of benign prostatic epithelium. Prostate 2013, 73, 476-488.

57. Vetvicka, V.; Vetvickova, J.; Benes, P. Role of enzymatically inactive procathepsin D in lung cancer. Anticancer Res. 2004, 24, 2739-2743.

58. Nazeer, T.; Malfetano, J.H.; Rosano, T.G.; Ross, J.S. Correlation of tumor cytosol Cathepsin D with differentiation and invasiveness of endometrial adenocarcinoma. Am. J. Clin. Pathol. 1992, 97, 764-769.

59. Baekelandt, M.; Holm, R.; Trope, C.G.; Nesland, J.M.; Kristensen, G.B. The significance of metastasis-related factors cathepsin-D and nm23 in advanced ovarian cancer. Ann. Oncol. 1999, 10, 1335-1341.

60. Ferrandina, G.; Scambia, G.; Fagotti, A.; D’Agostino, G.; Benedetti Panici, P.; Carbone, A.; Mancuso, S. Immunoradiometric and immunohistochemical analysis of cathepsin D in ovarian cancer: Lack of association with clinical outcome. Br. J. Cancer 1998, 78, 1645-1652.

61. Henzen-Logmans, S.C.; Fieret, E.J.; Berns, E.M.; van der Burg, M.E.; Klijn, J.G.; Foekens, J.A. Ki-67 staining in benign, borderline, malignant primary and metastatic ovarian tumors: Correlation with steroid receptors, epidermal-growth-factor receptor and cathepsin D. Int. J. Cancer 1994, 57, $468-472$.

62. Losch, A.; Schindl, M.; Kohlberger, P.; Lahodny, J.; Breitenecker, G.; Horvat, R.; Birner, P. Cathepsin D in ovarian cancer: Prognostic value and correlation with p53 expression and microvessel density. Gynecol. Oncol. 2004, 92, 545-552.

63. Chai, Y.; Wu, W.; Zhou, C.; Zhou, J. The potential prognostic value of Cathepsin D protein in serous ovarian cancer. Arch. Gynecol. Obstet. 2012, 286, 465-471.

64. Winiarski, B.K.; Cope, N.; Alexander, M.; Pilling, L.C.; Warren, S.; Acheson, N.; Gutowski, N.J.; Whatmore, J.L. Clinical relevance of increased endothelial and mesothelial expression of proangiogenic proteases and vegfa in the omentum of patients with metastatic ovarian high-grade serous carcinoma. Transl. Oncol. 2014, 7, 267-276.

65. Brouillet, J.P.; Dufour, F.; Lemamy, G.; Garcia, M.; Schlup, N.; Grenier, J.; Mani, J.C.; Rochefort, H. Increased cathepsin D level in the serum of patients with metastatic breast carcinoma detected with a specific pro-cathepsin D immunoassay. Cancer 1997, 79, 2132-2136.

66. Vetvicka, V.; Vektvickova, J.; Fusek, M. Effect of human procathepsin D on proliferation of human cell lines. Cancer Lett. 1994, 79, 131-135.

67. Ollinger, K. Inhibition of cathepsin D prevents free-radical-induced apoptosis in rat cardiomyocytes. Arch. Biochem. Biophys. 2000, 373, 346-351.

68. Kagedal, K.; Johansson, U.; Ollinger, K. The lysosomal protease cathepsin D mediates apoptosis induced by oxidative stress. FASEB J. 2001, 15, 1592-1594. 
69. Heinrich, M.; Neumeyer, J.; Jakob, M.; Hallas, C.; Tchikov, V.; Winoto-Morbach, S.; Wickel, M.; Schneider-Brachert, W.; Trauzold, A.; Hethke, A.; et al. Cathepsin D links TNF-induced acid sphingomyelinase to Bid-mediated caspase-9 and -3 activation. Cell Death Differ. 2004, 11, 550-563.

70. Blomgran, R.; Zheng, L.; Stendahl, O. Cathepsin-cleaved bid promotes apoptosis in human neutrophils via oxidative stress-induced lysosomal membrane permeabilization. J. Leukoc. Biol. 2007, 81, 1213-1223.

71. Johansson, A.C.; Steen, H.; Ollinger, K.; Roberg, K. Cathepsin d mediates cytochrome C release and caspase activation in human fibroblast apoptosis induced by staurosporine. Cell Death Differ. 2003, 10, 1253-1259.

72. Zuzarte-Luis, V.; Montero, J.A.; Torre-Perez, N.; Garcia-Porrero, J.A.; Hurle, J.M. Cathepsin D gene expression outlines the areas of physiological cell death during embryonic development. Dev. Dyn. 2007, 236, 880-885.

73. Zuzarte-Luis, V.; Montero, J.A.; Kawakami, Y.; Izpisua-Belmonte, J.C.; Hurle, J.M. Lysosomal cathepsins in embryonic programmed cell death. Dev. Biol. 2007, 301, 205-217.

74. Beaujouin, M.; Baghdiguian, S.; Glondu-Lassis, M.; Berchem, G.; Liaudet-Coopman, E. Overexpression of both catalytically active and -inactive cathepsin D by cancer cells enhances apoptosis-dependent chemo-sensitivity. Oncogene 2006, 25, 1967-1973.

75. Tardy, C.; Tyynela, J.; Hasilik, A.; Levade, T.; Andrieu-Abadie, N. Stress-induced apoptosis is impaired in cells with a lysosomal targeting defect but is not affected in cells synthesizing a catalytically inactive cathepsin D. Cell Death Differ. 2003, 10, 1090-1100.

76. Ohri, S.S.; Vashishta, A.; Proctor, M.; Fusek, M.; Vetvicka, V. The propeptide of cathepsin D increases proliferation, invasion and metastasis of breast cancer cells. Int. J. Oncol. 2008, 32, 491-498.

77. Briozzo, P.; Morisset, M.; Capony, F.; Rougeot, C.; Rochefort, H. In vitro degradation of extracellular matrix with Mr 52,000 cathepsin D secreted by breast cancer cells. Cancer Res. 1988, 48, 3688-3692.

78. Westley, B.R.; May, F.E. Cathepsin D and breast cancer. Eur. J. Cancer 1996, 32, 15-24.

79. Crowe, D.L.; Shuler, C.F. Regulation of tumor cell invasion by extracellular matrix. Histol. Histopathol. 1999, 14, 665-671.

80. Laurent-Matha, V.; Maruani-Herrmann, S.; Prebois, C.; Beaujouin, M.; Glondu, M.; Noel, A.; Alvarez-Gonzalez, M.L.; Blacher, S.; Coopman, P.; Baghdiguian, S.; et al. Catalytically inactive human cathepsin D triggers fibroblast invasive growth. J. Cell Biol. 2005, 168, 489-499.

81. Garcia, M.; Derocq, D.; Pujol, P.; Rochefort, H. Overexpression of transfected cathepsin D in transformed cells increases their malignant phenotype and metastatic potency. Oncogene 1990, 5, 1809-1814.

82. Liaudet, E.; Garcia, M.; Rochefort, H. Cathepsin D maturation and its stimulatory effect on metastasis are prevented by addition of kdel retention signal. Oncogene 1994, 9, 1145-1154.

83. Liaudet, E.; Derocq, D.; Rochefort, H.; Garcia, M. Transfected cathepsin D stimulates high density cancer cell growth by inactivating secreted growth inhibitors. Cell Growth Differ. 1995, 6, 1045-1052. 
84. Glondu, M.; Coopman, P.; Laurent-Matha, V.; Garcia, M.; Rochefort, H.; Liaudet-Coopman, E. A mutated cathepsin-D devoid of its catalytic activity stimulates the growth of cancer cells. Oncogene 2001, 20, 6920-6929.

85. Berchem, G.; Glondu, M.; Gleizes, M.; Brouillet, J.P.; Vignon, F.; Garcia, M.; Liaudet-Coopman, E. Cathepsin-D affects multiple tumor progression steps in vivo: Proliferation, angiogenesis and apoptosis. Oncogene 2002, 21, 5951-5955.

86. Fusek, M.; Vetvicka, V. Mitogenic function of human procathepsin D: The role of the propeptide. Biochem. J. 1994, 303, 775-780.

87. Vetvicka, V.; Vetvickova, J.; Fusek, M. Effect of procathepsin D and its activation peptide on prostate cancer cells. Cancer Lett. 1998, 129, 55-59.

88. Vetvicka, V.; Vetvickova, J.; Fusek, M. Anti-human procathepsin D activation peptide antibodies inhibit breast cancer development. Breast Cancer Res. Treat. 1999, 57, 261-269.

89. Vetvicka, V.; Vetvickova, J.; Hilgert, I.; Voburka, Z.; Fusek, M. Analysis of the interaction of procathepsin D activation peptide with breast cancer cells. Int. J. Cancer 1997, 73, 403-409.

90. Rochefort, H.; Capony, F.; Garcia, M.; Cavailles, V.; Freiss, G.; Chambon, M.; Morisset, M.; Vignon, F. Estrogen-induced lysosomal proteases secreted by breast cancer cells: A role in carcinogenesis? J. Cell. Biochem. 1987, 35, 17-29.

91. Vignon, F.; Capony, F.; Chambon, M.; Freiss, G.; Garcia, M.; Rochefort, H. Autocrine growth stimulation of the Mcf 7 breast cancer cells by the estrogen-regulated $52 \mathrm{k}$ protein. Endocrinology 1986, 118, 1537-1545.

92. Wolf, M.; Clark-Lewis, I.; Buri, C.; Langen, H.; Lis, M.; Mazzucchelli, L. Cathepsin D specifically cleaves the chemokines macrophage inflammatory protein-1 alpha, macrophage inflammatory protein-1 beta, and SLC that are expressed in human breast cancer. Am. J. Pathol. 2003, 162, 1183-1190.

93. Hu, L.; Roth, J.M.; Brooks, P.; Luty, J.; Karpatkin, S. Thrombin up-regulates cathepsin D which enhances angiogenesis, growth, and metastasis. Cancer Res. 2008, 68, 4666-4673.

94. Kirschke, H.; Langner, J.; Wiederanders, B.; Ansorge, S.; Bohley, P. Cathepsin 1. A new proteinase from rat-liver lysosomes. Eur. J. Biochem. 1977, 74, 293-301.

95. Kominami, E.; Ueno, T.; Muno, D.; Katunuma, N. The selective role of cathepsins B and D in the lysosomal degradation of endogenous and exogenous proteins. FEBS Lett. 1991, 287, 189-192.

96. Kominami, E.; Tsukahara, T.; Hara, K.; Katunuma, N. Biosyntheses and processing of lysosomal cysteine proteinases in rat macrophages. FEBS Lett. 1988, 231, 225-228.

97. Nishimura, Y.; Furuno, K.; Kato, K. Biosynthesis and processing of lysosomal cathepsin L in primary cultures of rat hepatocytes. Arch. Biochem. Biophys. 1988, 263, 107-116.

98. Towatari, T.; Katunuma, N. Amino acid sequence of rat liver cathepsin L. FEBS Lett. 1988, 236, $57-61$.

99. Ishidoh, K.; Towatari, T.; Imajoh, S.; Kawasaki, H.; Kominami, E.; Katunuma, N.; Suzuki, K. Molecular cloning and sequencing of cDNA for rat cathepsin L. FEBS Lett. 1987, 223, 69-73.

100. Dong, J.M.; Sahagian, G.G. Basis for low affinity binding of a lysosomal cysteine protease to the cation-independent mannose 6-phosphate receptor. J. Biol. Chem. 1990, 265, 4210-4217. 
101. Ishidoh, K.; Muno, D.; Sato, N.; Kominami, E. Molecular cloning of cDNA for rat cathepsin C. Cathepsin C, a cysteine proteinase with an extremely long propeptide. J. Biol. Chem. 1991, 266, 16312-16317.

102. Ritonja, A.; Popovic, T.; Kotnik, M.; Machleidt, W.; Turk, V. Amino acid sequences of the human kidney cathepsins H and L. FEBS Lett. 1988, 228, 341-345.

103. Mason, R.W.; Gal, S.; Gottesman, M.M. The identification of the major excreted protein (MEP) from a transformed mouse fibroblast cell line as a catalytically active precursor form of cathepsin $\mathrm{L}$. Biochem. J. 1987, 248, 449-454.

104. Mason, R.W.; Massey, S.D. Surface activation of pro-cathepsin L. Biochem. Biophys. Res. Commun. 1992, 189, 1659-1666.

105. Nakagawa, T.; Roth, W.; Wong, P.; Nelson, A.; Farr, A.; Deussing, J.; Villadangos, J.A.; Ploegh, H.; Peters, C.; Rudensky, A.Y. Cathepsin 1: Critical role in II degradation and CD4 T cell selection in the thymus. Science 1998, 280, 450-453.

106. Hsieh, C.S.; deRoos, P.; Honey, K.; Beers, C.; Rudensky, A.Y. A role for cathepsin L and cathepsin $\mathrm{S}$ in peptide generation for Mhc class II presentation. J. Immunol. 2002, 168, 2618-2625.

107. Roth, W.; Deussing, J.; Botchkarev, V.A.; Pauly-Evers, M.; Saftig, P.; Hafner, A.; Schmidt, P.; Schmahl, W.; Scherer, J.; Anton-Lamprecht, I.; et al. Cathepsin 1 deficiency as molecular defect of furless: Hyperproliferation of keratinocytes and pertubation of hair follicle cycling. FASEB J. 2000, 14, 2075-2086.

108. Potts, W.; Bowyer, J.; Jones, H.; Tucker, D.; Freemont, A.J.; Millest, A.; Martin, C.; Vernon, W.; Neerunjun, D.; Slynn, G.; et al. Cathepsin 1-deficient mice exhibit abnormal skin and bone development and show increased resistance to osteoporosis following ovariectomy. Int. J. Exp. Pathol. 2004, 85, 85-96.

109. Von Figura, K.; Hasilik, A. Lysosomal enzymes and their receptors. Annu. Rev. Biochem. 1986, $55,167-193$.

110. Dahms, N.M.; Lobel, P.; Kornfeld, S. Mannose 6-phosphate receptors and lysosomal enzyme targeting. J. Biol. Chem. 1989, 264, 12115-12118.

111. Dong, J.M.; Prence, E.M.; Sahagian, G.G. Mechanism for selective secretion of a lysosomal protease by transformed mouse fibroblasts. J. Biol. Chem. 1989, 264, 7377-7383.

112. Prence, E.M.; Dong, J.M.; Sahagian, G.G. Modulation of the transport of a lysosomal enzyme by PDGF. J. Cell Biol. 1990, 110, 319-326.

113. Achkar, C.; Gong, Q.M.; Frankfater, A.; Bajkowski, A.S. Differences in targeting and secretion of cathepsins B and L by BALB/3T3 fibroblasts and Moloney murine sarcoma virus-transformed BALB/3T3 fibroblasts. J. Biol. Chem. 1990, 265, 13650-13654.

114. Cuvier, C.; Jang, A.; Hill, R.P. Exposure to hypoxia, glucose starvation and acidosis: Effect on invasive capacity of murine tumor cells and correlation with cathepsin $(\mathrm{L}+\mathrm{B})$ secretion. Clin. Exp. Metastasis 1997, 15, 19-25.

115. Xu, X.; Greenland, J.; Baluk, P.; Adams, A.; Bose, O.; McDonald, D.M.; Caughey, G.H. Cathepsin L protects mice from mycoplasmal infection and is essential for airway lymphangiogenesis. Am. J. Respir. Cell Mol. Biol. 2013, 49, 437-444.

116. Maciewicz, R.A.; Wotton, S.F. Degradation of cartilage matrix components by the cysteine proteinases, cathepsins B and L. Biomed. Biochim. Acta 1991, 50, 561-564. 
117. Nguyen, Q.; Mort, J.S.; Roughley, P.J. Cartilage proteoglycan aggregate is degraded more extensively by cathepsin L than by cathepsin B. Biochem. J. 1990, 266, 569-573.

118. Maciewicz, R.A.; Wotton, S.F.; Etherington, D.J.; Duance, V.C. Susceptibility of the cartilage collagens types II, IX and XI to degradation by the cysteine proteinases, cathepsins B and L. FEBS Lett. 1990, 269, 189-193.

119. Nosaka, A.Y.; Kanaori, K.; Teno, N.; Togame, H.; Inaoka, T.; Takai, M.; Kokubo, T. Conformational studies on the specific cleavage site of type i collagen ( $\alpha-1)$ fragment (157-192) by cathepsins K and L by proton Nmr spectroscopy. Bioorg. Med. Chem. 1999, 7, 375-379.

120. Mason, R.W.; Johnson, D.A.; Barrett, A.J.; Chapman, H.A. Elastinolytic activity of human cathepsin L. Biochem. J. 1986, 233, 925-927.

121. Ishidoh, K.; Kominami, E. Procathepsin L degrades extracellular matrix proteins in the presence of glycosaminoglycans in vitro. Biochem. Biophys. Res. Commun. 1995, 217, 624-631.

122. Nishida, Y.; Kohno, K.; Kawamata, T.; Morimitsu, K.; Kuwano, M.; Miyakawa, I. Increased cathepsin L levels in serum in some patients with ovarian cancer: Comparison with CA125 and CA72-4. Gynecol. Oncol. 1995, 56, 357-361.

123. Zhang, W.; Wang, S.; Wang, Q.; Yang, Z.; Pan, Z.; Li, L. Overexpression of cysteine cathepsin L is a marker of invasion and metastasis in ovarian cancer. Oncol. Rep. 2014, 31, 1334-1342.

124. Zhang, L.; Wei, L.; Shen, G.; He, B.; Gong, W.; Min, N.; Zhang, L.; Duan, Y.; Xie, J.; Luo, H.; et al. Cathepsin 1 is involved in proliferation and invasion of ovarian cancer cells. Mol. Med. Rep. 2015, 11, 468-474.

125. Chambers, A.F.; Colella, R.; Denhardt, D.T.; Wilson, S.M. Increased expression of cathepsins L and $\mathrm{B}$ and decreased activity of their inhibitors in metastatic, ras-transformed NIH 3T3 cells. Mol. Carcinog. 1992, 5, 238-245.

126. Frade, R.; Rodrigues-Lima, F.; Huang, S.; Xie, K.; Guillaume, N.; Bar-Eli, M. Procathepsin-L, a proteinase that cleaves human $\mathrm{C} 3$ (the third component of complement), confers high tumorigenic and metastatic properties to human melanoma cells. Cancer Res. 1998, 58, 2733-2736.

127. Ishidoh, K.; Kominami, E. Gene regulation and extracellular functions of procathepsin L. Biol. Chem. 1998, 379, 131-135.

128. Yang, Z.; Cox, J.L. Cathepsin L increases invasion and migration of B16 melanoma. Cancer Cell Int. 2007, doi:10.1186/1475-2867-7-8.

129. Gocheva, V.; Zeng, W.; Ke, D.; Klimstra, D.; Reinheckel, T.; Peters, C.; Hanahan, D.; Joyce, J.A. Distinct roles for cysteine cathepsin genes in multistage tumorigenesis. Genes Dev. 2006, 20, 543-556.

130. Chung, J.H.; Im, E.K.; Jin, T.W.; Lee, S.M.; Kim, S.H.; Choi, E.Y.; Shin, M.J.; Lee, K.H.; Jang, Y. Cathepsin 1 derived from skeletal muscle cells transfected with bfgf promotes endothelial cell migration. Exp. Mol. Med. 2011, 43, 179-188.

131. Felbor, U.; Dreier, L.; Bryant, R.A.; Ploegh, H.L.; Olsen, B.R.; Mothes, W. Secreted cathepsin L generates endostatin from collagen XVIII. EMBO J. 2000, 19, 1187-1194.

132. Urbich, C.; Heeschen, C.; Aicher, A.; Sasaki, K.; Bruhl, T.; Farhadi, M.R.; Vajkoczy, P.; Hofmann, W.K.; Peters, C.; Pennacchio, L.A.; et al. Cathepsin L is required for endothelial progenitor cell-induced neovascularization. Nat. Med. 2005, 11, 206-213. 
133. Fiebiger, E.; Maehr, R.; Villadangos, J.; Weber, E.; Erickson, A.; Bikoff, E.; Ploegh, H.L.; Lennon-Dumenil, A.M. Invariant chain controls the activity of extracellular cathepsin L. J. Exp. Med. 2002, 196, 1263-1269.

134. Shimada, N.; Ohno-Matsui, K.; Iseki, S.; Koike, M.; Uchiyama, Y.; Wang, J.; Yoshida, T.; Sato, T.; Peters, C.; Mochizuki, M.; et al. Cathepsin L in bone marrow-derived cells is required for retinal and choroidal neovascularization. Am. J. Pathol. 2010, 176, 2571-2580.

(C) 2015 by the authors; licensee MDPI, Basel, Switzerland. This article is an open access article distributed under the terms and conditions of the Creative Commons Attribution license (http://creativecommons.org/licenses/by/4.0/). 